\title{
PARECERISTAS 2017*
}

\section{Anabela Cruz-Santos Santos \\ Universidade do Minho, Portugal \\ Ada Augusta Celestino Bezerra \\ Universidade Tiradentes, Brasil \\ Adair Mendes Nacarato \\ Universidade São Francisco, Brasil}

\section{Adilson Dalben}

Universidade Estadual de Campinas, Brasil

\section{Adolfo Ignacio Calderón}

Pontifícia Universidade de Campinas, Brasil

\section{Adriana Thoma}

Universidade Federal do Rio Grande do Sul, Brasil

\section{Agueda Bernardete Bitencourt}

Universidade Estadual de Campinas, Brasil

\section{Alda Junqueira Marin}

Pontifícia Universidade Católica de São Paulo, Brasil

\section{Alex Sander da Silva}

Universidade do Extremo Sul Catarinense, Brasil

\section{Alexandre Filordi de Carvalho}

Universidade Federal de São Paulo, Brasil

\section{Alexandre Simão Freitas}

Universidade Federal de Pernambuco, Brasil

\section{Alexsandro da Silva}

Universidade Federal de Pernambuco, Brasil

\section{Aliciene Machado Cordeiro}

Universidade da Região de Joinville, Brasil

\section{Alzira Mitz Bernardes Guarany}

Universidade Federal do Rio de Janeiro, Brasil

\section{Ana Elisa Spaolonzi Queiroz Assis}

Universidade Estadual de Campinas, Brasil

\section{Ana Ivenicki}

Universidade Federal do Rio de Janeiro, Brasil

\section{Ana Karina Brenner}

Universidade do Estado do Rio de Janeiro, Brasil

Ana Lucia Goulart de Faria

Universidade Estadual de Campinas, Brasil

\section{Ana María Botella Nicolás}

Universidad de Valencia, Espanha

Ana Maria Ferreira da Costa Monteiro

Universidade Federal do Rio de Janeiro, Brasil

Ana Maria Fonseca Almeida

Universidade Estadual de Campinas, Brasil

\section{Ana Maria Saul}

Pontifícia Universidade Católica de São Paulo, Brasil

\section{Ana Paula dos Santos de Sá}

Universidade Estadual de Campinas, Brasil

\section{Ana Paula Solino Bastos}

Universidade Estadual de Santa Cruz, Brasil

\section{André Augusto Diniz Lira}

Universidade Federal de Campina Grande, Brasil

\section{Ane Carine Meurer}

Universidade Federal de Santa Maria, Brasil

\section{Angela Cristina Fortes Iório}

Secretaria de Estado de Educação do Rio de Janeiro, Brasil

Angela Maria Franco Martins Coelho de Paiva Balça

Universidade de Évora, Portugal

\section{Antonio Alvaro Soares Zuin}

Universidade Federal de São Carlos, Brasil

António Augusto Moreira

Universidade de Aveiro, Portugal

\section{Antonio Carlos Rodrigues de Amorim}

Universidade Estadual de Campinas, Brasil

\section{Antonio Santos Jr}

Instituto Federal de Educação, Ciência e Tecnologia de Rondônia, Brasil

\footnotetext{
*A lista de pareceristas 2017 é resultado do relatório de avaliações concluídas no período
} de $1 \% / 01 / 2017$ a $31 / 12 / 2017$. 


\author{
Aruna Noal Correa \\ Universidade Federal de Santa Maria, Brasil \\ Áurea Maria Guimarães \\ Universidade Estadual de Campinas, Brasil \\ Bernardete Angelina Gatti \\ Fundação Carlos Chagas, Brasil \\ Candido Alberto da Costa Gomes \\ Universidade Católica de Brasília, Brasil

\section{Carlos Bauer} \\ Universidade Nove de Julho, Brasil

\section{Carlos Bernardo Skliar} \\ Facultad Latinoamericana de Ciencias Sociales, Argentina

\section{Carlos Frederico Bernardo Loureiro} \\ Universidade Federal do Rio de Janeiro, Brasil

\section{Carlos Herold Junior} \\ Universidade Estadual de Maringá, Brasil

\section{Carlos Roberto Jamil Cury \\ Pontifícia Universidade Católica de Minas Gerais, Brasil}

\section{Carmen Lúcia Soares} \\ Universidade Estadual de Campinas, Brasil

\section{Carolina de Roig Catini} \\ Universidade Estadual de Campinas, Brasil

\section{Caroline Jaques Cubas} \\ Universidade do Estado de Santa Catarina, Brasil
}

\section{Cecília Maria Aldigueri Goulart}

Universidade Federal Fluminense, Brasil

\section{Celia Maria Haas}

Universidade Cidade de São Paulo, Brasil

\section{Célia Regina Otranto}

Universidade Federal Rural do Rio de Janeiro, Brasil

\section{Célia Tanajura Machado}

Universidade do Estado da Bahia, Brasil

\section{Celso do Prado Ferraz de Carvalbo}

Universidade Nove de Julho, Brasil

\section{Cláudia Beatriz de Castro Nascimento Ometto \\ Universidade Estadual de Campinas, Brasil}

\section{Claudia Maria Costa Alves}

Universidade Federal Fluminense, Brasil

\section{Claudia Maria de Lima}

Universidade Estadual Paulista, Brasil

\section{Cláudia Ribeiro Bellochio}

Universidade Federal de Santa Maria, Brasil

Cláudio Almir Dalbosco

Universidade de Passo Fundo, Brasil

Cláudio José de Oliveira

Universidade de Santa Cruz do Sul, Brasil

\section{Claudio Roberto Baptista}

Universidade Federal do Rio Grande do Sul, Brasil

\section{Cristiane Machado}

Universidade Estadual de Campinas, Brasil

\section{Dalila Andrade Oliveira}

Universidade Federal de Minas Gerais, Brasil

\section{Damião Bezerra Oliveira}

Universidade Federal do Pará, Brasil

\section{Daniel Cavalcanti de Albuquerque Lemos}

Universidade Federal de Juiz de Fora, Brasil

\section{Daniervelin Renata Marques Pereira}

Universidade Federal do Triângulo Mineiro, Brasil

\section{Danilo Romeu Streck}

Universidade do Vale do Rio dos Sinos, Brasil

\section{Dante Henrique Moura}

Instituto Federal de Educação, Ciência e Tecnologia do Rio Grande do Norte, Brasil

\section{David Antonio da Costa}

Universidade Federal de Santa Catarina, Brasil

\section{David Mesquita Costa}

Centro Universitário Adventista de São Paulo, Brasil

\section{Débora Quetti Marques de Souza}

Universidade de Pernambuco, Brasil

\section{Delmary Vasconcelos de Abreu}

Universidade de Brasília, Brasil

\section{Denise Bessa Léda}

Universidade Federal do Maranhão, Brasil

\section{Diana Gomes da Silva Cerdeira}

Universidade do Estado do Rio de Janeiro, Brasil

\section{Diego Carlos Zanella}

Centro Universitário Franciscano, Brasil

Diego de Carvalho

Universidade de São Paulo, Brasil

\section{Dilma Antunes Silva}

Universidade Federal de São Paulo, Brasil 
Dirce Djanira Pacheco e Zan

Universidade Estadual de Campinas, Brasil

\section{Dulce Regina Baggio Osinski}

Universidade Federal do Paraná, Brasil

\section{Edméa Oliveira dos Santos}

Universidade do Estado do Rio de Janeiro, Brasil

\section{Edna Maria Querido de Oliveira Chamon}

Universidade de Taubaté, Brasil

\section{Edna Mendonça Oliveira Queiroz}

Universidade Federal de Goiás, Brasil

\section{Eduardo José Manzini}

Universidade Estadual Paulista, Brasil

\section{Edwin Herazo Acevedo}

Instituto de Investigacion Del Comportamiento

Humano, Colombia

\section{Eimard Gomes Antunes do Nascimento}

Universidade de Aveiro, Portugal

\section{Elane Nardotto Rios Cabral}

Instituto Federal de Educação, Ciência e Tecnologia da Bahia, Brasil

\section{Elenilton Vieira Godoy}

Universidade Federal do Paraná, Brasil

\section{Elenita Pinheiro de Queiroz Silva}

Universidade Federal de Uberlândia, Brasil

\section{Eliana Merlin Deganutti de Barros}

Universidade Estadual do Norte do Paraná, Brasil

\section{Elianda Figueiredo Arantes Tiballi}

Pontifícia Universidade Católica de Goiás, Brasil

\section{Eliane Maria Monteiro da Fonte}

Universidade Federal de Pernambuco, Brasil

\section{Érica Speglich}

Universidade Estadual de Campinas, Brasil

\section{Fabiana de Amorim Marcello}

Universidade Federal do Rio Grande do Sul, Brasil

\section{Fábio Dal-Soto \\ Universidade de Cruz Alta, Brasil}

\section{Fabrício Oliveira da Silva}

Universidade do Estado da Bahia, Brasil

\section{Fernanda Leopoldina Viana}

Universidade do Minho, Portugal
Fernando Augusto Starepravo

Universidade Estadual de Maringá, Brasil

\section{Fernando José Martins}

Universidade do Oeste do Paraná, Brasil

Flávia da Silva Ferreira Asbahr

Universidade Estadual Paulista Júlio de Mesquita Filho, Brasil

\section{Flavio Henrique Albert Brayner}

Universidade Federal de Pernambuco, Brasil

\section{Frederico Kauffmann Barbosa}

Universidade de Sorocaba, Brasil

\section{Gabriela Guarnieri de Campos Tebet}

Universidade Estadual de Campinas, Brasil

\section{Gabriela Portugal}

Universidade de Aveiro, Portugal

\section{Gabriela Tannús-Valadão}

Universidade Federal de São Carlos, Brasil

\section{Gilberto Icle}

Universidade Federal do Rio Grande do Sul, Brasil

\section{Gionara Tauchen}

Universidade Federal do Rio Grande, Brasil

\section{Giovane Saionara Ramos}

Universidade Federal Rural do Rio de Janeiro, Brasil

\section{Guilherme Welter Wendt}

University of London, Grã-Bretanha

\section{Heike Schmitz}

Universidade Federal de Sergipe, Brasil

\section{Heloísa Andreia de Matos Lins}

Universidade Estadual de Campinas, Brasil

\section{Ilse Abegg}

Universidade Federal de Santa Maria, Brasil

\section{Inajara de Salles Viana Neves}

Universidade Federal de Minas Gerais, Brasil

\section{Inês Barbosa de Oliveira}

Universidade do Estado do Rio de Janeiro, Brasil

\section{Inocêncio Fernandes Balieiro Filho}

Universidade Estadual Paulista, Brasil

\section{Isabel Cafezeiro}

Universidade Federal Fluminense, Brasil

\section{Isabel Cristina Moura Carvalho}

Pontifícia Universidade Católica do Rio Grande do Sul, Brasil 


\section{Isabela Rosália Lima Araújo}

Universidade Federal de Sergipe, Brasil

\section{Ivana Maria Schnitman}

Universidade Federal do Sul da Bahia, Brasil

\section{Ivanilde Apoluceno de Oliveira}

Universidade do Estado do Pará, Brasil

\section{Jaime Giolo}

Universidade Federal da Fronteira Sul, Brasil

\section{Jaime Valim Mansan}

Escola SESI de Ensino Médio Montenegro/RS, Brasil

\section{Janete Magalhães Carvalbo}

Universidade Federal do Espírito Santo, Brasil

\section{Jaqueline Moll}

Universidade Federal do Rio Grande do Sul, Brasil

\section{Javier Collado-Ruano}

Universidad Nacional de Educación, Equador

\section{Jean Henrique Costa}

Universidade do Estado do Rio Grande do Norte, Brasil

\section{Jefferson Mainardes}

Universidade Estadual de Ponta Grossa, Brasil

\section{João Colares da Mota Neto}

Universidade do Estado do Pará, Brasil

\section{João Ferreira de Oliveira}

Universidade Federal de Goiás, Brasil

\section{João Henrique Suanno}

Universidade Estadual de Goiás, Brasil

\section{João Menelau Paraskeva}

National Louis University, Estados Unidos

\section{Jorge Luiz da Cunha}

Universidade Federal de Santa Maria, Brasil

\section{Jorge Luiz da Silva}

Universidade de São Paulo, Brasil

\section{José Alberto Lencastre}

Universidade do Minho, Portugal

\section{Jose Armando Valente}

Universidade Estadual de Campinas, Brasil

\section{José Carlos Rothen}

Universidade Federal de São Carlos, Brasil

\section{José Francisco Chicon}

Universidade Federal do Espírito Santo, Brasil

\section{José Geraldo Silveira Bueno}

Pontifícia Universidade Católica de São Paulo, Brasil

\section{José Gonçalves Gondra}

Universidade do Estado do Rio de Janeiro, Brasil

José Licínio Backes

Universidade Católica Dom Bosco, Brasil

\section{José Pedro Boufleuer}

Universidade Regional do Noroeste do Estado do Rio Grande do Sul, Brasil

\section{José Pereira Peixoto Filho}

Universidade do Estado de Minas Gerais, Brasil

Joyce Wassem

Universidade Estadual de Campinas, Brasil

\section{Karinne Oliveira Coelho}

Instituto Federal de Alagoas, Brasil

\section{Karla Schuck Saraiva}

Universidade Luterana do Brasil, Brasil

\section{Katia Morosov Alonso}

Universidade Federal de Mato Grosso, Brasil

\section{Katya Mitsuko Zuquim Braghini}

Pontifícia Universidade Católica de São Paulo, Brasil

\section{Kazumi Munakata}

Pontifícia Universidade Católica de São Paulo, Brasil

\section{Laura Cristina Vieira Pizzi}

Universidade Federal de Alagoas, Brasil

\section{Lia Raquel Oliveira}

Universidade do Minho, Portugal

\section{Lilian Cristine Ribeiro Nascimento}

Universidade Estadual de Campinas, Brasil

\section{Lílian de Aragão Bastos do Valle}

Universidade do Estado do Rio de Janeiro, Brasil

\section{Lino Castellani Filho}

Universidade Estadual de Campinas, Brasil

\section{Lizete Maria Orquiza Carvalho}

Universidade Estadual Paulista, Brasil

\section{Lorene Figueiredo de Oliveira}

Universidade Federal Fluminense, Brasil

Lúcia Graça Cruz Domingues Amante

Universidade Aberta, Portugal

\section{Luciana Esmeralda Ostetto}

Universidade Federal Fluminense, Brasil 
Luciana Marta Del-Ben

Universidade Federal do Rio Grande do Sul, Brasil

\section{Luciane Ferreira de Oliveira Bonaldo}

Universidade Anhembi Morumbi, Brasil

Luciane Maria Schlindwein

Universidade Federal de Santa Catarina, Brasil

\section{Luciano Ferreira Silva}

Universidade Nove de Julho, Brasil

\section{Luciano Nascimento Corsino}

Instituto Federal de Educação, Ciência e Tecnologia do Rio Grande do Sul, Brasil

\section{Lucídio Bianchetti}

Universidade Federal de Santa Catarina, Brasil

\section{Luciene Fontão}

Secretaria Municipal de Educação de Florianópolis, Brasil

\section{Lucinalva Andrade Ataide de Almeida}

Universidade Federal de Pernambuco, Brasil

\section{Luis Fernando Cerri}

Universidade Estadual de Ponta Grossa, Brasil

\section{Luís Filipe Barbeiro}

Instituto Politécnico de Leiria, Portugal

\section{Luis Henrique Sacchi dos Santos}

Universidade Federal do Rio Grande do Sul, Brasil

\section{Luis Henrique Sommer}

Universidade Federal do Rio Grande do Sul, Brasil

\section{Luiz Carlos Gil Esteves}

Universidade Federal do Estado do Rio de Janeiro, Brasil

\section{Luiz Cláudio da Silva Câmara}

Universidade Federal do Rio de Janeiro, Brasil

\section{Magali Aparecida Silvestre}

Universidade Federal de São Paulo, Brasil

\section{Manuel Lázaro Pulido}

Universidade Católica Portuguesa, Portugal

\section{Marcelo Andrade \\ Pontifícia Universidade Católica do Rio de Janeiro, Brasil (in memorian)}

\section{Marcelo de Andrade Pereira}

Universidade Federal de Santa Maria, Brasil

\section{Marcelo Pinheiro Cigales}

Universidade Federal de Santa Catarina, Brasil

\section{Marcelo Siqueira Maia Vinagre Mocarzel}

Instituto Maia Vinagre, Brasil

Márcia Denise Pletsch

Universidade Federal Rural do Rio de Janeiro, Brasil

\section{Márcia Serra Ferreira}

Universidade Federal do Rio de Janeiro, Brasil

Marcia Soares Alvarenga

Universidade do Estado do Rio de Janeiro, Brasil

\section{Márcia Souza Hobold}

Universidade da Região de Joinville, Brasil

\section{Marco Antonio Leandro Barzano}

Universidade Estadual de Feira de Santana, Brasil

\section{Marcos Augusto Souza Rodrigues da Silva}

Universidade do Vale do Paraíba, Brasil

\section{Marcus Levy Bencostta}

Universidade Federal do Paraná, Brasil

\section{Marcus Vinícius Cunba}

Universidade de São Paulo, Brasil

\section{Marcus Vinícius Medeiros Pereira}

Universidade Federal de Juiz de Fora, Brasil

\section{Marek Tesar}

University of Auckland, Nova Zelandia

\section{Maria Abádia da Silva}

Universidade de Brasília, Brasil

\section{Maria Alfredo Moreira}

Universidade do Minho, Portugal

\section{Maria Amélia Rosário Santoro Franco}

Universidade Católica de Santos, Brasil

\section{Maria Auxiliadora Moreira dos Santos Schmidt}

Universidade Federal do Paraná, Brasil

\section{Maria Carla Corrochano}

Universidade Federal de São Carlos, Brasil

\section{Maria Carmo Lacerda Peixoto}

Universidade Federal de Minas Gerais, Brasil

\section{Maria Cecília Lorea Leite}

Universidade Federal de Pelotas, Brasil

\section{Maria Celina Piazza Recena}

Universidade Federal de Mato Grosso do Sul, Brasil

\section{Maria Constantina Caputo}

Universidade Federal da Bahia, Brasil 
Maria Cristina de Carvalho Cascelli de Azevedo Universidade de Brasília, Brasil

Maria Cristina Soares de Gouvêa

Universidade Federal de Minas Gerais, Brasil

Maria da Conceição Ferrer Botelho Sgadari Passeggi Universidade Federal do Rio Grande do Norte, Brasil

\section{Maria das Graças Gonçalves Vieira Guerra} Universidade Federal da Paraíba, Brasil

\section{Maria de Fátima Barbosa Abdalla}

Universidade Católica de Santos, Brasil

\section{Maria do Rosário Figueiredo Tripodi}

Universidade Federal de Ouro Preto, Brasil

\section{Maria dos Remédios de Brito}

Universidade Federal do Pará, Brasi

Maria Elizabeth Bianconcini Pinto de Almeida

Pontifícia Universidade Católica de São Paulo, Brasil

\section{Maria Fátima Barbosa Abdalla}

Universidade Católica de Santos, Brasil

\section{Maria Helena Camara Bastos}

Pontifícia Universidade Católica do Rio Grande do Sul, Brasil

\section{Maria Helena Michels}

Universidade Federal de Santa Catarina, Brasil

Maria Idalina Lourido Figueiredo dos Santos Universidade de Coimbra, Portugal

\section{Maria Isabel Ramalho Ortigão}

Universidade do Estado do Rio de Janeiro, Brasil

\section{Maria Letícia Barros Pedroso Nascimento}

Universidade de São Paulo, Brasil

\section{Maria Lúcia Rodrigues Muller}

Universidade Federal de Mato Grosso, Brasil

\section{Maria Luiza de Santana Lombas}

Coordenação de Aperfeiçoamento de Pessoal de Nível Superior, Brasil

\section{Maria Luiza Süssekind Verissimo Cinelli \\ Universidade Federal do Estado do Rio de Janeiro, Brasil}

\section{Maria Margarida Machado}

Universidade Federal de Goiás, Brasil

Maria Remédios Brito

Universidade Federal do Pará, Brasil
Maria Teresa Ceron Trevisol

Universidade do Oeste de Santa Catarina, Brasil

Maria Teresa Cortes Zavala

Universidad Michoacana de San Nicolás de Hidalgo, México

\section{Maria Teresa Santos Cunha}

Universidade do Estado de Santa Catarina, Brasil

Mariana Aranba de Souza

Universidade de Taubaté, Brasil

María-Pilar Cáceres Reche

Universidad de Granada, Espanha

Marilda Oliveira de Oliveira

Universidade Federal de Santa Maria, Brasil

\section{Marilia Gouvea de Miranda}

Universidade Federal de Goiás, Brasil

\section{Mário Luiz Ferrari Nunes}

Universidade Estadual de Campinas, Brasil

\section{Marisa Bittar}

Universidade Federal de São Carlos, Brasil

Marlécio Maknamara da Silva Cunha

Universidade Federal da Bahia, Brasil

Marli Elisa Dalmaso Afonso de André

Pontifícia Universidade Católica de São Paulo, Brasil

\section{Marly Amarilha}

Universidade Federal do Rio Grande do Norte, Brasil

\section{Marta Maria Chagas de Carvalho}

Universidade de São Paulo, Brasil

\section{Mary Sandra Carlotto}

Universidade do Vale do Rio dos Sinos, Brasil

\section{Mauricio Ernica}

Universidade Estadual de Campinas, Brasil

\section{Maximiliano Durán}

Universidad de Buenos Aires, Argentina

\section{Messias Holanda Dieb}

Universidade Federal do Ceará, Brasil

Miria Gomes de Oliveira

Universidade Federal de Minas Gerais, Brasil

Miriam Aparecida Graciano de Souza Pan

Universidade Federal do Paraná, Brasil

\section{Miriam Soares Leite}

Universidade do Estado do Rio de Janeiro, Brasil 
Mirian Jorge Warde

Universidade Federal de São Paulo, Brasil

Monica Fantin

Universidade Federal de Santa Catarina, Brasil

Monique Andries Nogueira

Universidade Federal do Rio de Janeiro, Brasil

Múcio Luiz Banja Fernandes

Universidade de Pernambuco, Brasil

Mylene Nogueira Teixeira

Universidade Estadual do Norte Fluminense Darcy

Ribeiro, Brasil

Nadia Bigarella

Universidade Católica Dom Bosco, Brasil

\section{Nadia Gaiofatto Gonçalves}

Universidade Federal do Paraná, Brasil

\section{Nadja Mara Amilibia Hermann}

Universidade Federal do Rio Grande do Sul, Brasil

Neil Franco Pereira de Almeida

Universidade Federal de Juiz de Fora, Brasil

\section{Nelson Cardoso Amaral}

Universidade Federal de Goiás, Brasil

\section{Nerli Nonato Ribeiro Mori}

Universidade Estadual de Maringá, Brasil

\section{Nesdete Mesquita Corrêa}

Universidade Federal de Mato Grosso do Sul, Brasil

\section{Neusa Chaves Batista}

Universidade Federal do Rio Grande do Sul, Brasil

\section{Norma Sandra Almeida Ferreira}

Universidade Estadual de Campinas, Brasil

\section{Núbia Pereira Paiva}

Universidade Estadual do Sudoeste da Bahia, Brasil

\section{Ofélia Agoglia}

Universidad Nacional de Cuyo - Argentina

\section{Olgaíes Cabral Maués}

Universidade Federal do Pará, Brasil

\section{Pablo Silva Machado Bispo dos Santos}

Universidade Federal Fluminense, Brasil

\section{Patrícia da Conceição Fantinel}

Universidade Federal de Pelotas, Brasil

\section{Patricio Alfonso Landaeta Mardones}

Universidad de Playa Ancha, Chile

\section{Paula da Silva Pereira}

Universidade do Minho, Portugal

Paulo Gerson Rodrigues Stefanello

Universidade Federal de São Carlos, Brasil

Paulo Renato Bernardes Nobre

Universidade de Coimbra, Portugal

Paulo Sérgio Garcia

Universidade de São Paulo, Brasil

\section{Philippe Pomier Layrargues}

Universidade de Brasília, Brasil

\section{Ramon de Oliveira}

Universidade Federal de Pernambuco, Brasil

\section{Raquel Gonçalves Salgado}

Universidade Federal de Mato Grosso, Brasil

\section{Raquel Goulart Barreto}

Universidade do Estado do Rio de Janeiro, Brasil

\section{Regiane Helena Bertagna}

Universidade Estadual Paulista, Brasil

\section{Renato José de Oliveira}

Universidade Federal do Rio de Janeiro, Brasil

\section{Rhenan Ferraz de Jesus}

Instituto Federal Farroupilha, Brasil

\section{Rita de Cassia Prazeres Frangella}

Universidade do Estado do Rio de Janeiro, Brasil

\section{Rocío Chao Fernández}

Universidade da Coruña, Espanha

\section{Rodrigo Alves dos Santos}

Centro Federal de Educação Tecnológica de Minas

Gerais, Brasil

\section{Rodrigo Barchi}

Universidade de Sorocaba, Brasil

\section{Rodrigo Lema Del Rio Martins}

Universidade Federal do Espírito Santo, Brasil

\section{Rodrigo Manoel Dias da Silva}

Universidade do Vale do Rio dos Sinos, Brasil

\section{Rogério Rodrigues}

Universidade Federal de Itajubá, Brasil

\section{Ronaldo Marcos de Lima Araújo}

Universidade Federal do Pará, Brasil

\section{Roney Polato de Castro}

Universidade Federal de Juiz de Fora, Brasil 


\section{Rosa Maria Bueno Fischer}

Universidade Federal do Rio Grande do Sul, Brasil

Rosa Vásquez Recio

Universidade de Cádiz, Espanha

Rosalia Maria Duarte

Pontifícia Universidade Católica do Rio de Janeiro, Brasil

\section{Rosângela Tenório de Carvalho}

Universidade Federal de Pernambuco, Brasil

Rosemeire Reis da Silva

Universidade Federal de Alagoas, Brasil

Rosivalda dos Santos Barreto

Secretaria da Educação do Estado da Bahia, Brasil

Salomão Antônio Mufarrej Hage

Universidade Federal do Pará, Brasi

Samuel Mendonça

Pontifícia Universidade Católica de Campinas, Brasil

Sandra Eli Sartoreto de Oliveira Martins

Universidade Estadual Paulista Júlio de Mesquita Filho, Brasil

\section{Sandra Regina Simonis Richter}

Universidade de Santa Cruz do Sul, Brasil

\section{Sérgio Paulino Abranches}

Universidade Federal de Pernambuco, Brasil

\section{Shaula Maíra Vicentini de Sampaio}

Universidade Federal Fluminense, Brasil

\section{Sidelmar Alves da Silva Kunz}

Universidade Federal de Goiás, Brasil

Simone de Oliveira da Silva Santos

Faculdade Cenecista de Itaboraí, Brasil

Simone Lucena Ferreira

Universidade Federal de Sergipe, Brasil

\section{Simone Torres Evangelista}

Universidade Federal Fluminense, Brasil

\section{Solange Estanislau dos Santos}

Universidade Federal de Alagoas, Brasil

\section{Sonia Barbosa Camargo Igliori}

Pontifícia Universidade Católica de São Paulo, Brasil

\section{Sônia Cristina Soares Dias Vermelho}

Universidade Federal do Rio de Janeiro, Brasil
Sonia Maria da Silva Junqueira

Universidade Federal do Pampa, Brasil

\section{Sueli Salva}

Universidade Federal de Santa Maria, Brasil

\section{Susana Beatriz Sacavino}

Pontifícia Universidade Católica do Rio de Janeiro, Brasil

\section{Suzana dos Santos dos Santos Gomes}

Universidade Federal de Minas Gerais, Brasil

\section{Suzana dos Santos Gomes}

Universidade Federal de Minas Gerais, Brasil

Suzani Cassiani

Universidade Federal de Santa Catarina, Brasil

\section{Tatiana de Souza Camargo}

Universidade Federal do Rio Grande do Sul, Brasil

Thelma Maria de Moura Bergamo

Tecnológico do Instituto Federal Goiano, Brasil

Thiago Ranniery Moreira de Oliveira

Universidade Federal do Rio de Janeiro, Brasil

Timothy Denis Ireland

Universidade Federal da Paraíba, Brasil

\section{Valmor Rhoden}

Universidade Federal do Pampa, Brasil

Vera Maria Nigro de Souza Placco

Pontifícia Universidade Católica de São Paulo, Brasil

Virgínia Cecília da Rocha Louzada

Universidade do Estado do Rio de Janeiro, Brasil

Viviane Castro Camozzato

Universidade Estadual do Rio Grande do Sul, Brasil

Wenceslao Machado de Oliveira Junior

Universidade Estadual de Campinas, Brasil

Wilma Baía Coelho

Universidade Federal do Pará, Brasil

\section{Wivian Weller}

Universidade de Brasília, Brasil

Zulma Elizabete de Freitas Madruga

Universidade Estadual de Santa Cruz, Brasil

Zulmira Newlands Borges

Universidade Federal de Santa Maria, Brasil 